\title{
Deriving the Glutamate Clearance Time Course from Transporter Currents in CA1 Hippocampal Astrocytes: Transmitter Uptake Gets Faster during Development
}

\author{
Jeffrey S. Diamond \\ Synaptic Physiology Unit, National Institute of Neurological Disorders and Stroke, National Institutes of Health, Bethesda, Maryland 20892-3701
}

\begin{abstract}
At many excitatory synapses, the neurotransmitter glutamate diffuses beyond the synaptic cleft to activate extrasynaptic targets. The extent and impact of such transmitter "spillover" on the processing capacity of neuronal networks are unclear, in part because it remains unknown how far transmitter diffuses from its point of release before being removed from the extracellular space by high-affinity glutamate transporters. Synaptically activated, transporter-mediated currents (STCs) recorded in hippocampal astrocytes provide an experimental measure of glutamate uptake, but the time course of the STC may be shaped, or "filtered," by other factors and therefore not represent a direct indication of clearance rate. Here, STCs were recorded from astrocytes in rat hippocampal slices under conditions in which uptake capacity was reduced and the STC decay reflected a slowed rate of glutamate clearance. The temporal characteristics of the filtering mechanisms were extracted from these responses, and the glutamate clearance time course in control conditions was derived. The results indicate that glutamate can be cleared from the extrasynaptic space within $1 \mathrm{~ms}$. Clearance is fastest in adult neuropil, corresponding to a developmental increase in glial transporter expression. Synaptically released glutamate is taken up at the same rate as glutamate released via flash photolysis, indicating that the spatial location of transporters relative to the site of glutamate release does not affect the time course of clearance. Slower clearance in young animals would permit glutamate to diffuse greater distances, indicating a particularly important role for extrasynaptic receptors early in development.
\end{abstract}

Key words: diffusion; spillover; hippocampus; transporter; uptake; synaptic

\section{Introduction}

Fast, excitatory signaling in the CNS appears mediated primarily by receptors in the postsynaptic density, directly apposed to the presynaptic active zone. At many synapses, however, glutamate also activates receptors and transporters outside the synaptic cleft (Bergles et al., 1999). These targets often regulate synaptic efficacy and plasticity, and so it is important to determine the extent of their activation under different conditions and at different developmental stages. A first step to this goal is to determine experimentally how long and how far glutamate diffuses from its point of release before it is removed from the extracellular space by high-affinity transporters.

An accurate measure of the rapid diffusion and clearance of glutamate requires a fast sensor that does not affect synaptically released transmitter before its uptake. Previous studies have shown that synaptically activated, glutamate transportermediated currents (STCs) can be recorded in CA1 astrocytes

Received Dec. 15, 2004; revised Feb. 3, 2005; accepted Feb. 7, 2005.

This work was supported by the National Institute of Neurological Disorders and Stroke Intramural Research Program. I thank Joe Mindell, Nelson Spruston, and members of the laboratory for valuable discussions and John Isaac, Craig Jahr, Trisha Kalbaugh, and Joshua Singer for comments on this manuscript.

Correspondence should be addressed to Dr. Jeffrey S. Diamond, Synaptic Physiology Unit, National Institute of Neurological Disorders and Stroke, National Institutes of Health, Building 35, Room 3C-1000, 35 Convent Drive, Bethesda, MD 20892-3701. E-mail: diamondj@ninds.nih.gov.

D01:10.1523/JNEUROSCI.5125-04.2005

Copyright $\odot 2005$ Society for Neuroscience $\quad$ 0270-6474/05/252906-11\$15.00/0
(Bergles and Jahr, 1997). The STC time course appears to be shaped, to some extent, by the dynamics of glutamate clearance: the STC is slowed by transporter antagonists, which slow glutamate clearance by decreasing uptake capacity (Bergles and Jahr, 1997; Diamond and Jahr, 2000). Deriving the clearance rate from the STC time course is complicated, however, by the likely influence on the STC waveform of other factors such as asynchronous transmitter release and electrotonic filtering.

In this study, STCs were recorded from juvenile and adult rat hippocampal astrocytes at physiological temperatures to determine which factors influence the STC time course. The STC waveform was unaffected by changes in stimulus strength or release probability, confirming previous work (Diamond and Jahr, 2000). In addition, flash photolysis of caged glutamate elicited transporter currents [flash-evoked transporter currents (FTCs)] with a time course that was similar to that of STCs recorded in the same cells, regardless of how much glutamate was uncaged. These results indicate that the STC time course is unaffected by potential nonlinearities such as transporter saturation or spatially inhomogeneous transporter expression. STCs were significantly faster in adult astrocytes, suggesting that developmental increases in transporter expression (Furuta et al., 1997; Ullensvang et al., 1997) influence significantly the extent of extrasynaptic glutamate diffusion. The consequences of these developmental changes were examined more quantitatively using two methods to extract the time course of uptake from the STC waveform. The 
results indicate that synaptically released glutamate is taken up in the adult hippocampus with a time constant of $\sim 1 \mathrm{~ms}$, three to eight times faster than in the juvenile hippocampus. Evoked synaptic responses in CA1 pyramidal cells indicated that the developmental increase in uptake capacity reduces NMDA receptor (NMDAR) activation by glutamate spillover in the adult hippocampal neuropil.

\section{Materials and Methods}

Slice preparation and solutions. Hippocampal slices $(400 \mu \mathrm{m})$ were prepared from Sprague Dawley rats, as described previously (Sakmann and Stuart, 1995) and in accordance with National Institute of Neurological Disorders and Stroke Animal Care and Use Committee guidelines. Slices were prepared in ice-cold artificial CSF (ACSF) containing (in mM) 87 $\mathrm{NaCl}, 2.5 \mathrm{KCl}, 0.5 \mathrm{CaCl}_{2}, 7.0 \mathrm{MgCl}_{2}, 1.25 \mathrm{NaH}_{2} \mathrm{PO}_{4}, 25$ glucose, 75 sucrose, and $25 \mathrm{NaHCO}_{3}$ and stored in ACSF containing (in mM) 119 $\mathrm{NaCl}, 2.5 \mathrm{KCl}, 1.3 \mathrm{MgCl}_{2}, 2.5 \mathrm{CaCl}_{2}, 1 \mathrm{NaH}_{2} \mathrm{PO}_{4}, 26.2 \mathrm{NaHCO}_{3}$, and 11 glucose (both bubbled with a $95 \% \mathrm{O}_{2}-5 \% \mathrm{CO}_{2}$ mixture). After being cut on a vibratome (Leica, Nussloch, Germany), slices were stored at $34^{\circ} \mathrm{C}$ for $30 \mathrm{~min}$ and at room temperature for up to $7 \mathrm{~h}$ thereafter. For all experiments, extracellular solutions contained picrotoxin $(100 \mu \mathrm{M})$ and 6,7dinitroquinoxaline-2,3-dione (DNQX; $20 \mu \mathrm{M}$ ) to block GABA receptors and AMPA receptors (AMPARs), respectively. For recordings of transporter-mediated currents, extracellular solutions also contained $R, S$-3-(2-carboxypiperazin-4-yl)propyl-1-phosphonic acid (CPP; $5 \mu \mathrm{M}$ ) and (+)-5-methyl-10,11-dihydroxy-5 h dibenzo(a,d)cyclohepten-5,10imine $(\mathrm{MK}-801,5 \mu \mathrm{M})$ to block NMDARs and 8-cyclopentyl-1,3dimethylxanthine $(4 \mu \mathrm{M})$ to block $\mathrm{A}_{1}$ adenosine receptors and thereby increase release probability. Solutions were equilibrated with the $\mathrm{O}_{2}-$ $\mathrm{CO}_{2}$ mixture and superfused through the recording chamber at $\sim 2 \mathrm{ml} /$ $\mathrm{min}$. An in-line heater was used to warm the recording solution to $35 \pm$ $1^{\circ} \mathrm{C}$, as measured by a probe in the recording chamber. The recording pipette solution contained the following (in $\mathrm{mm}$ ): $120 \mathrm{~K}^{+}$methanesulfonate (for STCs) or $\mathrm{Cs}^{+}$methanesulfonate (for EPSCs), 10 EGTA, 20 HEPES, $2 \mathrm{MgATP}$, and $0.2 \mathrm{NaGTP}$, adjusted to $290 \mathrm{mOsm}$ with sucrose. Reagents were obtained from Sigma (St. Louis, MO), except for 4-methoxy-7-nitroindolinyl-caged L-glutamate (MNI-glutamate) and D,L-threo- $\beta$-benzyloxyaspartate (TBOA), which were obtained from Tocris Cookson (Ellisville, MO).

Electrophysiology. Slices were visualized on an upright fixed-stage microscope (Axioskop 2FS; Zeiss, Thornwood, NY) equipped with infrared-differential interference contrast optics. Recordings were made with an Axopatch 1D amplifier (Axon Instruments, Foster City, CA). Astrocytes in the CA1 stratum radiatum were identified by their small cell bodies, low $(<10 \mathrm{M} \Omega)$ input resistance, and very negative resting potential (approximately $-95 \mathrm{mV}$ ); the clamp potential was set at the astrocyte resting potential. Pyramidal neurons were voltage clamped at $+30 \mathrm{mV}$ (after correction for a junction potential of $-10 \mathrm{mV}$ ) to remove the $\mathrm{Mg}^{2+}$ block of NMDARs and inhibit postsynaptic glutamate transporters (Diamond, 2001). Data were acquired with a 16-bit analog-to-digital board (Instrutech, Port Washington, NY) controlled with software written in Igor Pro (WaveMetrics, Lake Oswego, OR) and analyzed with Igor Pro. Transporter currents were sampled at $10-50 \mathrm{kHz}$ and filtered at 5 $\mathrm{kHz}$. NMDAR EPSCs were sampled at $5 \mathrm{kHz}$ and filtered at $2 \mathrm{kHz}$. Patch electrodes (\#0010 glass; World Precision Instruments, Sarasota, FL) had tip resistances of 1.5-2.5 M $\Omega$. STCs and EPSCs were evoked with a bipolar stimulating electrode (115 $\mu \mathrm{m}$ pole spacing; Frederick Haer Company, Bowdoinham, ME) placed in the stratum radiatum 100-200 $\mu \mathrm{m}$ from the recorded cell. Access resistance was monitored throughout each experiment, was typically $<20 \mathrm{M} \Omega$ (measured by the peak of the charging current induced by a $1 \mathrm{mV}$ step), and was not compensated.

Flash photolysis of caged glutamate. MNI-glutamate was superfused through the recording chamber at a concentration of 100-200 $\mu \mathrm{M}$ and uncaged using a Xenon flash lamp (Flashmic; Rapp OptoElectronic, Hamburg, Germany) connected to the epi-illumination pathway of the microscope with a liquid light guide. The lamp output was set to maximum, and the entire back aperture of the $40 \times$ objective was illuminated, corresponding to a $250-\mu \mathrm{m}$-diameter spot in the plane
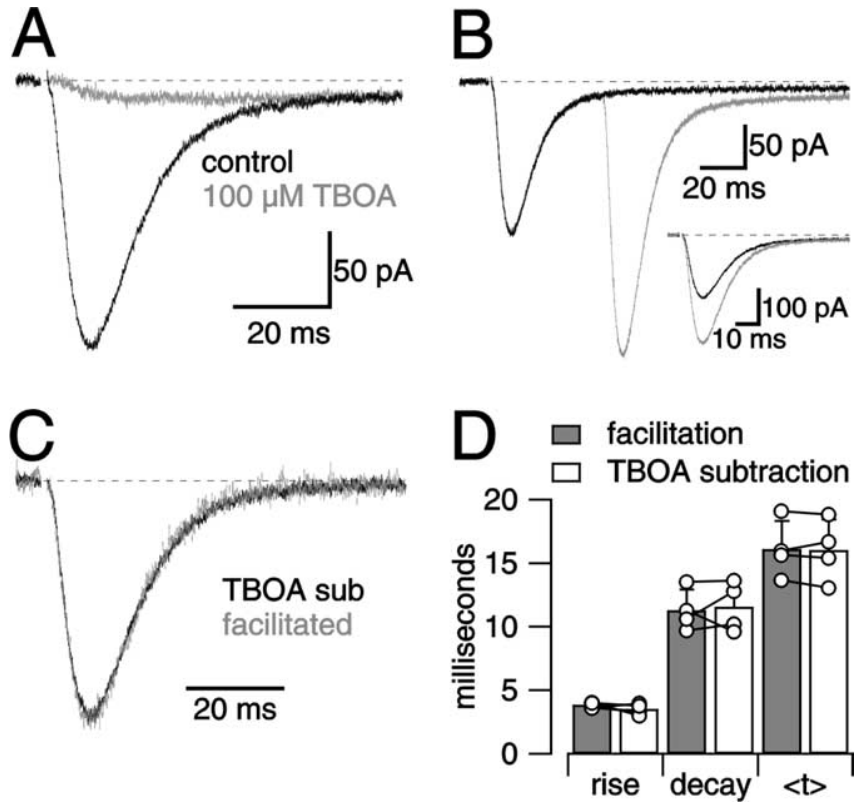

Figure 1. Isolation of the STC. $A$, Synaptic currents recorded in a P12 CA1 astrocyte in control solution (containing $G_{A B A} R$, AMPAR, and NMDAR antagonists; black trace) and in the additional presence of the competitive, nonsubstrate transporter antagonist TBOA (100 $\mu \mathrm{m}$; gray trace). TBOA blocked the transient component but not the sustained component, which reflects a slowly decaying potassium conductance. $\boldsymbol{B}$, Responses elicited by a single stimulus (black trace) or a pair of stimuli (50 ms interstimulus interval; gray trace). Inset, Subtraction of response to single stimulus from that to two stimuli isolates the facilitated response (gray trace), which is shifted in time for comparison with the single stimulus response (black trace). Note that the transporter-mediated component of the response facilitates more than the potassium current. Data are from the same cell as in $\boldsymbol{A}$. C, Comparison of TBOA-sensitive current in $\boldsymbol{A}$ (black trace) with the facilitated transporter current in $\boldsymbol{B}$ (gray trace; obtained by subtracting the single stimulus response from the facilitated response). $\boldsymbol{D}$, Summary data from four cells showing that TBOA subtraction (TBOA sub) and facilitation/subtraction yielded similar estimates of STC 20 $80 \%$ rise time, exponential decay time constant, and centroid. All data are from P12-P14 astrocytes; similar results were obtained in adult astrocytes.

of focus. Based on the manufacturer's test results, in the configuration used, the flash width at $50 \%$ of peak height was $330 \mu$ s and the flash was $99 \%$ complete in $680 \mu$ s.

Analysis. FTCs were isolated by subtracting the flashlamp-induced artifact recorded with the light path blocked. Responses recorded with the path blocked were similar to those recorded in the absence of MNIglutamate or in the presence of MNI-glutamate plus TBOA $(100 \mu \mathrm{M})$. STCs were isolated by subtraction of control responses either from responses in the presence of $100 \mu \mathrm{M}$ TBOA (Fig. 1A) or from averaged responses to paired stimuli by subtracting the response to the first stimulus from the facilitated response to the second stimulus (Fig. 1B,C). Either approach eliminated almost all of a very slowly decaying current thought to result from extracellular potassium accumulation (Fig. $1 A, B$ ) (Bergles and Jahr, 1997). The facilitation subtraction worked best, although in most cases a small residual potassium current remained, the amplitude of which constituted $2.1 \pm 1.2 \%$ of the STC amplitude in postnatal day $12(\mathrm{P} 12)$ to P14 cells $(n=13)$ and $4.1 \pm 2.6 \%$ of the STC amplitude in adults cells $(n=14)$. This residual current was approximated by a simple exponentially rising function scaled to the amplitude (A) of the residual current $100 \mathrm{~ms}$ after stimulation, as follows:

$$
A\left(1-\exp \left(\frac{-t}{\tau_{\text {rise }}}\right)\right)
$$

$\tau_{\text {rise }}$ was determined from a set of experiments in which the rising portion of the response in the presence of $100 \mu \mathrm{M}$ TBOA (Fig. $1 \mathrm{~A}$ ) was fit with an exponential function. $\tau_{\text {rise }}$ was $5.7 \pm 2.0 \mathrm{~ms}$ in P13 astrocytes $(n=4)$ and $3.3 \pm 1.2 \mathrm{~ms}$ in adult astrocytes $(n=5)$. This approximation waveform 
was then subtracted from the STC to eliminate the small residual potassium current.

For deconvolution analysis (see Figs. 5, 6), isolated transporter currents were fit to an exponentially rising and decaying function (Nielsen et al., 2004) to obtain a smooth waveform that closely approximated the STC (see Fig. $5 A, C$ ):

$$
f(t)=\mathrm{A}\left(1-\exp \left(-\frac{t-t_{0}}{\tau_{\mathrm{r}}}\right)\right)^{\mathrm{n}}\left[\exp \left(-\frac{t-t_{0}}{\tau_{\mathrm{f}}}\right)+0.05 \exp \left(-\frac{t-t_{0}}{\tau_{\mathrm{s}}}\right)\right]
$$

The last exponential term $\left(\tau_{\mathrm{s}}\right)$ stabilized the simultaneous fitting of the rising and decaying phases.

The centroid $(\langle t\rangle)$ of an FTC or STC was calculated as the ratio of the first moment of the waveform to its area (Bracewell, 2000):

$$
<t>=\frac{\int t f(t) d t}{\int f(t) d t}
$$

This calculation was straightforward when done graphically: The onset of the response was set to $t=0$, and each point in the response was multiplied by its corresponding time point. This waveform was integrated and divided by the integral of the response alone. The centroid, calculated in this manner, does not reflect delays in synaptic transmission because of action potential propagation along the Schaffer collateral axons.

Unless indicated otherwise, all data are reported as mean $\pm \mathrm{SD}$, and effects were deemed significant when $p<0.05$ in a Student's $t$ test.

Simulations. Transmitter diffusion was modeled in Matlab (MathWorks, Natick, MA) as a random walk of 5000 glutamate molecules from a point source within a $320-\mathrm{nm}$-diameter synaptic cleft (Ventura and Harris, 1999). At each time step $\Delta t$ (usually $1 \mu \mathrm{s}$ ), each glutamate molecule was displaced in each spatial dimension by a distance $r$ randomly selected from a normal distribution centered at zero [average $r^{2}=2 D \Delta t$ (Hille, 1984), where $D$ is the diffusion coefficient $]. D\left[0.76 \mu \mathrm{m}^{2} / \mathrm{ms}^{-1}\right.$ in aqueous solution at $25^{\circ} \mathrm{C}$ (Longsworth, 1953)] was reduced by twothirds to account for diffusion in extracellular fluid (Nielsen et al., 2004) and increased 1.3 -fold to account for the $35^{\circ} \mathrm{C}$ recording temperature (Hille, 1984). Varying $D$ had little effect on the simulated STC time course ( $D$ three times higher $\rightarrow\langle t\rangle_{\text {STC }} 3 \%$ lower, $D$ three times lower $\rightarrow\langle t\rangle_{\text {STC }} 11 \%$ higher; data not shown). Diffusion within the disk-like synaptic cleft was two-dimensional (Barbour and Hausser, 1997). Diffusion was three-dimensional through isotropic extrasynaptic space (extracellular volume fraction, 0.21 ), in which $D$ was reduced further to account for tortuosity $\left(D^{*}=D / \lambda^{2} ; \lambda=1.55\right)$ (Rusakov and Kullmann, 1998). To simulate the local barriers of the presynaptic bouton and postsynaptic spine, glutamate was excluded from the 320-nm-diameter sphere described by the cleft disk (see Fig. $8 \mathrm{~A}$ ). For simplicity, once a glutamate molecule left the cleft, it could not return. Simulations in which glutamate was permitted to return to the cleft were computationally much more expensive but yielded similar results (data not shown).

Glutamate transporters were modeled using a Markov representation of the EAAT2 subtype (Bergles et al., 2002), with several simplifying modifications. Extracellular binding events before glutamate binding (i.e., two sodium ions and a proton) were excluded, as was intracellular potassium binding. Unbound transporters needed only to bind glutamate and one sodium ion for translocation to occur, a reasonable assumption given the rapid binding rates of extracellular sodium and protons (Bergles et al., 2002). After translocation, the transporter unbound one sodium ion, the proton, glutamate, and two more sodium ions before returning to the unbound, outward-facing state at a rate corresponding to physiological measures of recovery (Bergles et al., 2002). Once all of the transported elements unbound on the intracellular side, the glutamate molecule was removed from the simulation. The simulated STC waveform (see Fig. $8 B$, inset) reflected the translocation step in the model [transition 8 in the study by Bergles et al. (2002)]: at each time step, the STC was incremented for each forward (inward) transition and decremented for each backward transition. To account for the temperature dependence of enzymatic reactions (Hille, 1984) and, in particular, transporter kinetics (Bergles and Jahr, 1998; Wadiche and Kavanaugh, 1998; Auger and Attwell, 2000), rates derived from experiments performed at $22-24^{\circ} \mathrm{C}$ (Bergles et al., 2002) were multiplied by 3 to approx- imate kinetics at $35^{\circ} \mathrm{C}$. Voltage-dependent rates were calculated with $V_{\mathrm{m}}=-95 \mathrm{mV}$.

Extracellular space was partitioned transparently into 10 -nm-thick concentric shells so that local transporter and glutamate concentrations could be determined. At each time step, the probability of binding to a transporter was determined independently for each glutamate molecule as follows. First, the EAAT2 glutamate-binding rate (Bergles et al., 2002) was multiplied by the time step and the glutamate concentration in the relevant shell to yield the probability of transporter binding. This number was multiplied by the number of transporter molecules in the shell, to give the number of transporters bound in the time step, and divided by the number of glutamate molecules in the shell to yield the probability that a particular glutamate molecule would bind. If binding occurred (i.e., if a random number between 0 and 1 was less than the binding probability), the number of free transporter molecules in the cell was decremented. Once bound, the glutamate molecule underwent probabilistic transitions during subsequent time steps through the Markov transporter scheme until it unbound on either side of the membrane. Because each modeled transporter was associated with an individual glutamate molecule, this approach required tracking no more than 5000 transporters and, consequently, allowed very high transporter concentrations to be simulated on a personal computer with $512 \mathrm{Mb}$ RAM.

NMDAR activation (see Fig. $8 D$ ) was simulated in ChanneLab (Synaptosoft, Decatur, GA) by driving a Markov model of an NMDAR (Lester and Jahr, 1992) with glutamate concentration waveforms generated by diffusion simulations. $P_{\text {activation }}($ see Fig. $8 D$ ) reflects the fraction of activated receptors (i.e., the fraction open divided by the maximal $P_{\mathrm{o}}$, which was 0.29 ). Transition rates in the NMDAR model were increased by a factor of 3 to account for the temperature dependence of NMDAR kinetics (Diamond, 2001) and simulate $35^{\circ} \mathrm{C}$.

\section{Results}

\section{Isolation of the STC}

During whole-cell patch recordings from astrocytes in the stratum radiatum of the $\mathrm{CA} 1$ region of hippocampal slices, AMPARs, NMDARs, and $\mathrm{GABA}_{\mathrm{A}}$ receptors $\left(\mathrm{GABA}_{\mathrm{A}} \mathrm{Rs}\right)$ were blocked with DNQX $(20 \mu \mathrm{M}), \mathrm{CPP}(5 \mu \mathrm{M}), \mathrm{MK}-801(5 \mu \mathrm{M})$, and picrotoxin $(100 \mu \mathrm{M})$. Under these conditions, stimulation of Schaffer collateral/commissural fibers elicited an inward current comprising fast and slow components (Fig. $1 A$ ). The transporter antagonist TBOA $(100 \mu \mathrm{M})$ blocked the fast component (Fig. $1 A)$, identifying it as an STC. The slower component is thought to reflect a potassium conductance caused by an increase in extracellular potassium after action potential propagation along the Schaffer collateral axons (Bergles and Jahr, 1997). To isolate the STC without using TBOA, pairs of stimuli were delivered at $50 \mathrm{~ms}$ intervals to elicit paired-pulse facilitation (Fig. $1 B$ ). Responses to stimulus pairs were interleaved with responses to single stimuli, and the facilitated response was isolated by subtracting the response to a single stimulus from that to a pair (Fig. $1 B$, inset). The STC component of the response facilitated but the potassium conductance did not (Fig. 1 $B$, inset), so subtraction of the facilitated response from the control response yielded a pure STC waveform that was indistinguishable in time course from the TBOAsensitive current (Fig. 1C,D) (see Materials and Methods). The STC time course was quantified by measuring its $20-80 \%$ rise time, exponential decay time constant, and centroid $(<t>$; see Materials and Methods), a measure of the temporal "center of mass" of the STC. The centroid reflects both the rise and decay kinetics of the STC and therefore serves as a useful measure of overall time course ( $<t>$ of an instantaneously rising, exponentially decaying waveform is equal to the exponential time constant). The centroid also plays a central role in analyses presented later in this report (see Fig. 7). Unless indicated otherwise, STCs described in subsequent experiments were isolated using the facilitation procedure. 

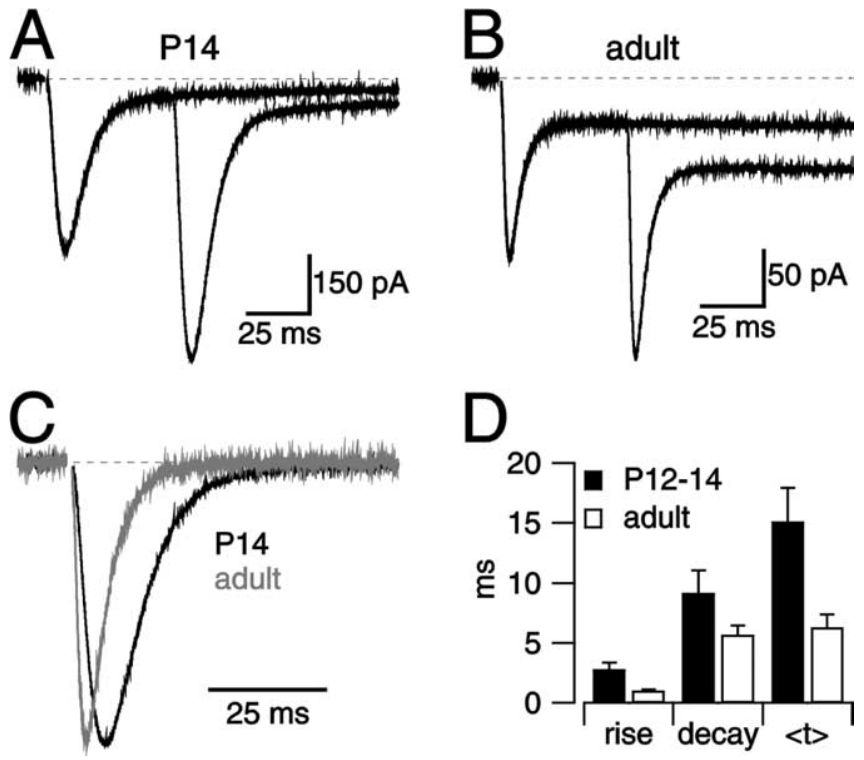

Figure 2. STCs get faster during development. $\boldsymbol{A}$, Synaptic currents in response to single and paired ( 50 ms interval) stimulation, recorded in a P14 astrocyte. $\boldsymbol{B}$, Responses as in $\boldsymbol{A}$, recorded from an adult astrocyte. $\boldsymbol{C}$, Facilitated STCs from $\boldsymbol{A}$ (black trace) and $\boldsymbol{B}$ (gray trace), normalized in amplitude to facilitate comparison of time course. $D$, Summary data showing that STCs recorded in adult astrocytes $(n=13)$ exhibited faster rise times, decay times, and centroids than STCs recorded in P12-P14 astrocytes $(n=13)$.

\section{STCs get faster during development}

Immunocytochemical studies indicate that glial glutamate transporter expression increases severalfold during development ( $\mathrm{Fu}-$ ruta et al., 1997; Ullensvang et al., 1997), suggesting that the rate of glutamate clearance may increase as well. Consistent with this prediction, the rise, decay, and $\langle t\rangle$ of STCs recorded in adult ( $>$ P60, $n=11$ ) astrocytes were significantly faster than those recorded in young (P12-P14, $n=10)$ astrocytes $(p \ll 0.001$ in all cases) (Fig. 2). The amplitude of the evoked potassium current was significantly larger, relative to the STC, in older astrocytes $(45 \pm 12 \% ; n=11)$ than in P12-P14 cells $(12 \pm 7 \% ; n=10$; P12-P14 vs adult; $p \ll 0.001)$. Analogous changes during the second postnatal week reflect a developmental increase in resting potassium conductance (Bergles and Jahr, 1997). In contrast, the increase in the evoked potassium conductance observed here between P12-P14 and adult was not associated with a change in input resistance (adult: $R_{\text {in }}=8.4 \pm 4.3 \mathrm{M} \Omega, n=11$; P12-P14: $\left.R_{\text {in }}=6.2 \pm 1.2 \mathrm{M} \Omega, n=10 ; p=0.13\right)$. The change, then, may reflect a developmental decrease in synaptic glutamate release (indicated by the STC) relative to the density of action potentials propagating through the CA1 neuropil (indicated by the evoked potassium conductance).

Ideally, the rate of glutamate clearance would be reflected directly in the decay kinetics of the STC. If this were the case, one would conclude that adult astrocytes take up glutamate about twice as rapidly as P12-P14 astrocytes ( $\sim 5 \mathrm{vs} \sim 10 \mathrm{~ms}$ ) (Fig. $2 D$ ) and that glutamate is permitted, even in the adult, to diffuse several micrometers from its point of release, much further than the average distance between neighboring synapses in CA1 (0.465 $\mu \mathrm{m})$ (Rusakov and Kullmann, 1998). However, if STC kinetics were slowed significantly by other factors such as electrotonic filtering and asynchronous transmitter release, the STC decay time course would represent an underestimate of the rate of glutamate clearance and, consequently, lead to an overestimate of glutamate diffusion distance. The relatively slow time course of the STC relative to transporter kinetics measured in excised patches (Wadiche and Kavanaugh, 1998; Bergles et al., 2002) and the expected kinetics of glutamate diffusion (Barbour, 2001) suggest that the STC likely reflects a filtered representation of glutamate clearance. The experiments described below were designed to determine the characteristics of this filtering to derive a more quantitative estimate of the glutamate clearance rate and, consequently, the distance of glutamate diffusion after its synaptic release.

\section{A linear filter distorts the STC}

We may consider the experimental system (the Schaffer collateral axons, the CA1 synapses and surrounding diffusion environment, the location and kinetics of glial glutamate transporters, the electrotonic properties the astrocytes and the characteristics of the recording system) as a series of filters that transforms a very brief $(100 \mu \mathrm{s})$ electrical stimulus into an STC that lasts tens of milliseconds (Fig. 1A). Some of these filters, such as those representing the time course of action potential propagation and the delay and asynchrony inherent in synaptic release, precede glutamate clearance and are unrelated to its dynamics but may, nonetheless, affect the time course over which glutamate is taken up during an STC. Others, such as those representing electrotonic filtering by the astrocyte membrane, follow clearance and do not affect the time course of uptake but affect the fidelity of the electrophysiological recording.

Previous work on P13-P15 astrocytes indicated that changing stimulus strength causes proportional changes in STC amplitude with no effects on the STC time course (Diamond and Jahr, 2000). This result suggests that the filters described above, as a group, behave in a linear manner, at least over the range of activation elicited by synaptic stimulation. This result was confirmed here in adult astrocytes (Fig. 3A): the charge transfer (Q) during STCs evoked by $30 \mu \mathrm{A}$ stimulation was proportionally less than that during STCs evoked by $100 \mu \mathrm{A}$ stimulation $\left(\mathrm{Q}_{30} / \mathrm{Q}_{100}=\right.$ $0.27 \pm 0.04 ; n=3)$, but there was no change in $20-80 \%$ rise time $(1.38 \pm 0.27$ vs $1.39 \pm 0.25 \mathrm{~ms})$, decay time constant (5.06 \pm 0.37 vs $4.94 \pm 0.31 \mathrm{~ms})$ or $\langle t\rangle(5.19 \pm 0.22$ vs $5.33 \pm 0.63 \mathrm{~ms})$ (Fig. $3 D)$. As another way to change the amplitude of the STC, the probability of release was increased by inducing paired-pulse facilitation (Figs. $1 B, 3 B$ ). This resulted in a $212 \pm 22 \%$ increase in $\mathrm{Q}(n=7)$ but had no effect on the $20-80 \%$ rise time $(1.12 \pm 0.19$ vs $1.04 \pm 0.12 \mathrm{~ms} ; p=0.15)$ or decay time $(5.06 \pm 0.37$ vs $4.94 \pm$ $0.31 \mathrm{~ms} ; p=0.61)$ and only a small increase in $\langle t>(6.12 \pm 1.34$ vs $6.44 \pm 1.27 \mathrm{~ms} ; p=0.015$ ) (Fig. $3 D$ ).

To measure glutamate uptake in a slice without the possible complications of axonal stimulation and synaptic release, transporter currents were recorded in response to flash photolysis of caged glutamate. Glutamate was presented to the slice in an instant (see Materials and Methods), and transporters removed it at their natural rate. Glutamate could not be removed quickly by diffusion, because the entire slice was superfused with caged glutamate and the light flash covered a $250-\mu \mathrm{m}$-diameter region of the slice, effectively eliminating local concentration gradients. FTCs were recorded while superfusion solution containing MNIglutamate was gradually washed into the chamber, yielding a series of responses to progressively higher glutamate concentrations (Fig. 3C). FTCs were blocked by $100 \mu \mathrm{M}$ TBOA (data not shown), indicating that they reflected activation of glutamate transporters. FTCs increased in amplitude as the MNI-glutamate concentration increased, but they exhibited consistent time courses (Fig. 3C,D).

The experiments described in Figure 3 indicate that the time 

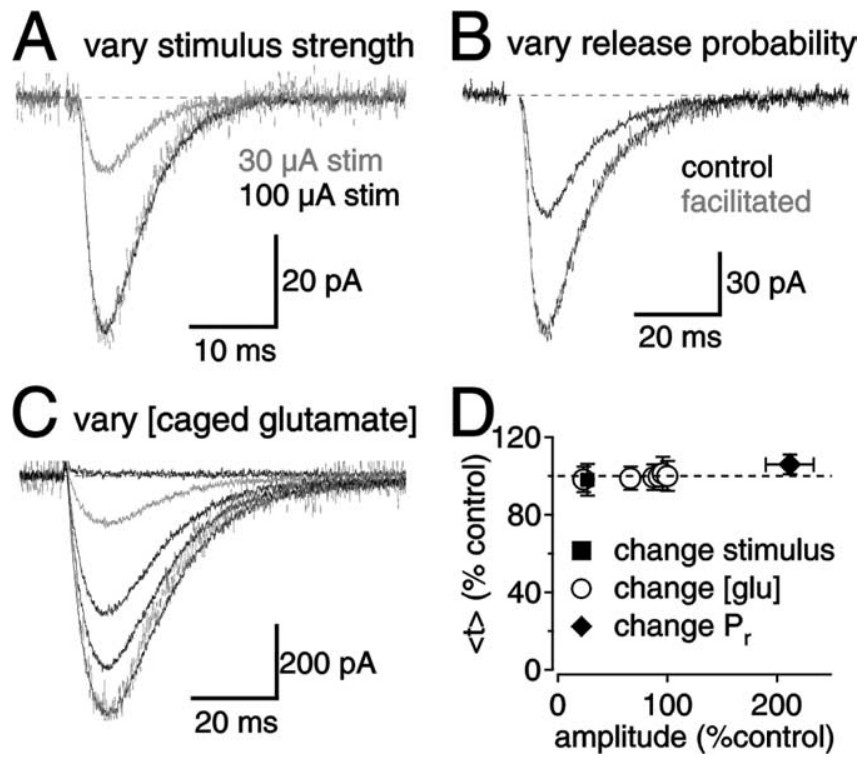

Figure 3. Varying stimulus-evoked glutamate release does not affect transporter current time course. A, STCs elicited by $30 \mu \mathrm{A}$ (gray trace) and $100 \mu \mathrm{A}$ (black trace) stimulation. The gray dotted trace shows a $30 \mu \mathrm{A}$ response scaled to the $100 \mu \mathrm{A}$ response. $\boldsymbol{B}$, STC elicited by a single stimulus (black trace) and the facilitated STC elicited by the second of a pair of stimuli (gray trace; single response subtracted from the isolated response to the second stimulus). The black dotted trace shows control response scaled to facilitated response for comparison of time course. The potassium current was removed by TBOA subtraction (see Fig. 1A). C, FTCs recorded while washing $100 \mu \mathrm{m}$ MNI-glutamate into the recording chamber. A small response (gray trace) was scaled (gray dotted trace) to the largest response to facilitate time course comparison. $\boldsymbol{D}$, Summary data showing that changing stimulus strength (squares; $n=4$ ), inducing facilitation (diamonds; $n=7$ ), or varying the concentration of caged glutamate (circles; $n=6$ ) had only small effects on the transporter current time course. All data are from adult astrocytes; similar results were obtained in P12-P14 astrocytes.

course of transporter-mediated currents is independent of the number of activated Schaffer axons, the release probability of activated synapses, or the substrate concentration presented by rapid photolysis of caged glutamate. The illustrated data were obtained in adult astrocytes; similar results were obtained in P12P14 astrocytes (data not shown). The consistency of the STC and FTC time course with changing stimulus strength and the proportional relationship between stimulus strength and STC amplitude (Fig. 3A) (Diamond and Jahr, 2000) indicate that the filters distorting the STC time course behave, to a good approximation, as a linear system over the ranges tested here.

\section{The spatial distribution of glutamate release does not affect the time course of uptake}

Glial glutamate transporters appear to cluster near synaptic connections (Chaudhry et al., 1995; Minelli et al., 2001; Zhou and Sutherland, 2004), suggesting that transporters are targeted specifically to optimize the uptake of synaptically released transmitter. It is possible, therefore, that glutamate escaping a perisynaptic transporter cluster could then diffuse into a region of lower transporter density. Moreover, glutamate released at extrasynaptic, "ectopic" sites (Matsui and Jahr, 2003) may be taken up more slowly than transmitter released within synaptic clefts. One might expect, therefore, that glutamate released via photolysis would be taken up more slowly than that released synaptically. To test this prediction, the time course of STCs and FTCs were compared in the same astrocytes (Fig. 4). Synaptic and flash stimulation were alternated in control solution and during the bath application of MNI-glutamate (100-200 $\mu \mathrm{M})$. The solution containing MNI-
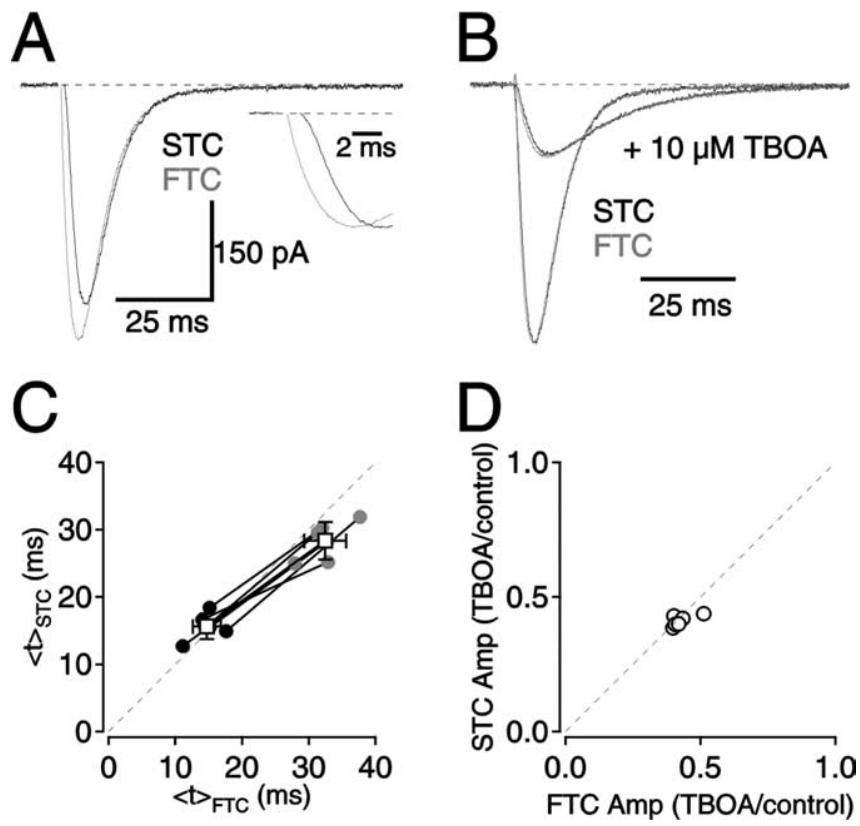

Figure 4. Synaptically and flash-evoked transporter currents exhibit similar time courses. $A$, Transporter currents elicited by synaptic stimulation (STC; black trace) and flash photolysis of caged glutamate (FTC; gray trace) recorded in the same astrocyte. Inset, STC and FTC scaled to the same amplitude to facilitate comparison of rise times. B, STC (black trace) and FTC (gray trace) from $A$, in control solution and in the presence of $10 \mu \mathrm{M}$ TBOA. STCs have been shifted in time $(1.4 \mathrm{~ms})$ to account for synaptic delay. Both STCs were scaled by the same amount to match the corresponding FTC amplitude. C, Comparison of the centroid of the STC and FTC in six astrocytes, in control (black circles) and in the presence of $10 \mu \mathrm{m}$ TBOA (gray circles). D, Fractional block of the STC amplitude by $10 \mu \mathrm{M}$ TBOA compared with the fractional block of the FTC amplitude (FTCAmp) in the same cell $(n=6)$. Data are from P12-P14 astrocytes; similar results were obtained in adult astrocytes.

glutamate induced a small but consistent slowing of the STC $(<t>$ increased from $13.21 \pm 1.3$ to $15.6 \pm 1.9 \mathrm{~ms} ; n=6 ; p=$ $0.003)$, consistent with a slight reduction in uptake capacity (Bergles and Jahr, 1997; Diamond and Jahr, 2000). To control for this small effect, FTCs were compared with STCs evoked in the presence of MNI-glutamate.

FTCs and STCs recorded in the same cell exhibited similar time courses (Fig. 4A). STCs were delayed 1-2 ms relative to the FTCs (Fig. $4 A$, inset), presumably because of the time required for action potentials to propagate from the site of stimulation to the presynaptic terminals. In addition, the rising phase of the STC exhibited a slightly more gradual rise (Fig. $4 A$, inset), probably because of the asynchrony of neurotransmitter release. When the STC was shifted in time to account for the synaptic delay, however, the STC and FTC superimposed very closely (Fig. 4B), suggesting that release asynchrony contributed insignificantly to the time course of the STC. In a group of cells from P12-P14 slices, the STC centroid (measured from the onset of the response) was not significantly different from that of the FTC $\left(\langle t\rangle_{\mathrm{STC}}, 15.6 \pm\right.$ $1.9 \mathrm{~ms} ;\langle t\rangle_{\text {FTC }}, 14.7 \pm 2.1 \mathrm{~ms} ; n=6 ; p=0.30$ ) (Fig. $4 \mathrm{C}$ ). A subsaturating concentration $(10 \mu \mathrm{M})$ of TBOA reduced the amplitude of STCs and FTCs to a similar extent (STC reduced to $41 \pm 2 \%$ of control; FTC reduced to $43 \pm 4 \%$ of control; $n=6$; $p=0.26$ ) (Fig. $4 D$ ), although the $\langle t\rangle_{\text {FTC }}$ was increased to a greater extent than was $\langle t\rangle_{\text {STC }}(223 \pm 16$ vs $183 \pm 22 \%$ of control; $n=6 ; p=0.017$ ) (Fig. $4 C$ ). To a first approximation, however, STCs and FTCs exhibited similar kinetics, suggesting that the relative spatial distribution of synaptically released glutamate and glial transporters is not a major determinant of the time course of uptake. 

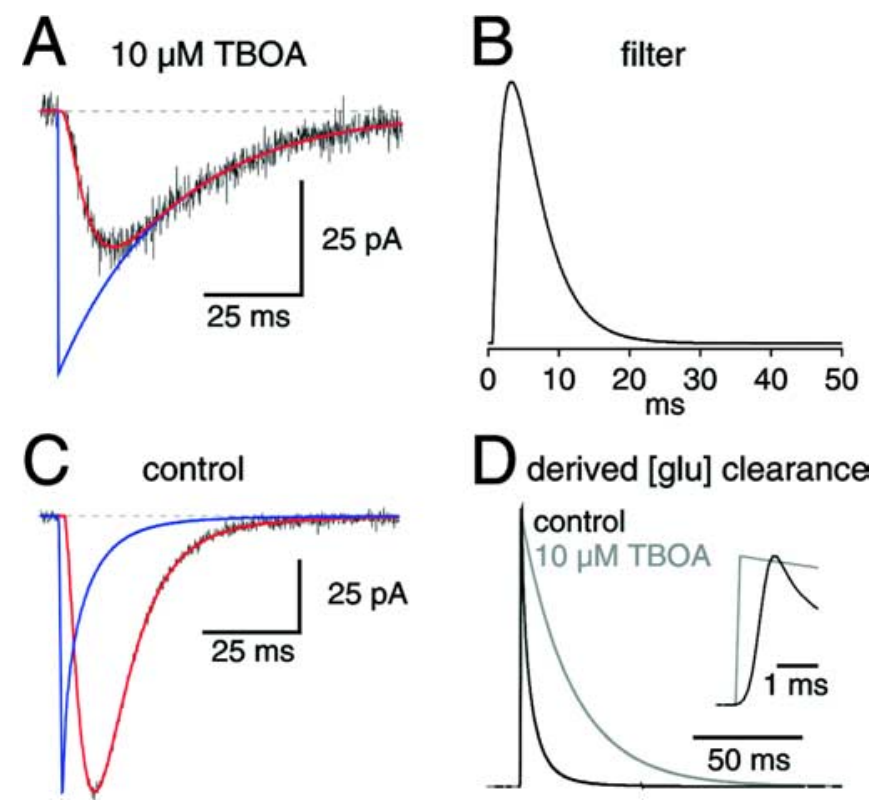

Figure 5. Deconvolving the filter to estimate glutamate clearance time in a P12 astrocyte. $A$, STC recorded in the presence of $10 \mu \mathrm{M}$ TBOA (black trace) and fit by a continuous function (red trace; see Materials and Methods). The ST ( decay was fit by a single-exponential function that was taken to indicate the slowed glutamate clearance rate in the presence of transporter antagonist (blue trace). $\boldsymbol{B}$, Temporal characteristics of filter derived from response in $\boldsymbol{A}$ by deconvolving the exponential clearance time course from the continuous fit to the STC in $10 \mu \mathrm{M} \mathrm{TBOA.}$ C, STC recorded from the same astrocyte as in $A$ in control conditions (black trace) and fit by a continuous function (red trace). The filter in $\boldsymbol{B}$ was deconvolved from the continuous function to derive an estimate of glutamate clearance in control conditions (blue trace). $\boldsymbol{D}$, Derived glutamate clearance time courses in control (black trace) and TBOA (gray trace), normalized and superimposed. Inset, Same traces on an expanded time scale for comparison of rising phases.

Deriving the time course of glutamate clearance from the STC The preceding experiments indicate that the mechanisms transforming an electrical stimulus into an STC behave like a linear system over the ranges tested, even if certain components are omitted (i.e., when glutamate is presented via photolysis). The STC, then, can be considered to represent the convolution of the time course of glutamate clearance (i.e., including transmitter diffusion and transporter kinetics, localization, and density) with a filter representing the remaining elements of the system (axon activation and propagation, release asynchrony, and electrotonic filtering). Under control conditions, the STC decay likely reflects a combination of glutamate clearance and other mechanisms. However, when uptake capacity is decreased by partial block of glutamate transporters, the STC decay is prolonged and dominated by the slow time course of glutamate clearance (Bergles and Jahr, 1997; Diamond and Jahr, 2000) (Fig. 3B). Under these conditions, STC amplitude remains proportional to stimulus strength, and the STC time course remains unaffected by response amplitude (Diamond and Jahr, 2000), suggesting that the system remains linear. Because both the STC and clearance time courses were known, the time course of the filter could be derived by deconvolving the clearance time course from the STC waveform. The time course of clearance in the presence of $10 \mu \mathrm{M}$ TBOA was determined by fitting the STC decay to a singleexponential function (Fig. $5 \mathrm{~A}$, blue line). In a group of recordings from young animals (P12-P14), the STC in $10 \mu \mathrm{M}$ TBOA decayed with a time constant of $28.4 \pm 6.4 \mathrm{~ms}(n=7)$. For simplicity, the rise of the clearance time course was made instantaneous, because the similarity in STC and FTC time course (Fig. 4) indicated that diffusion time from synaptic release sites to glial transporters
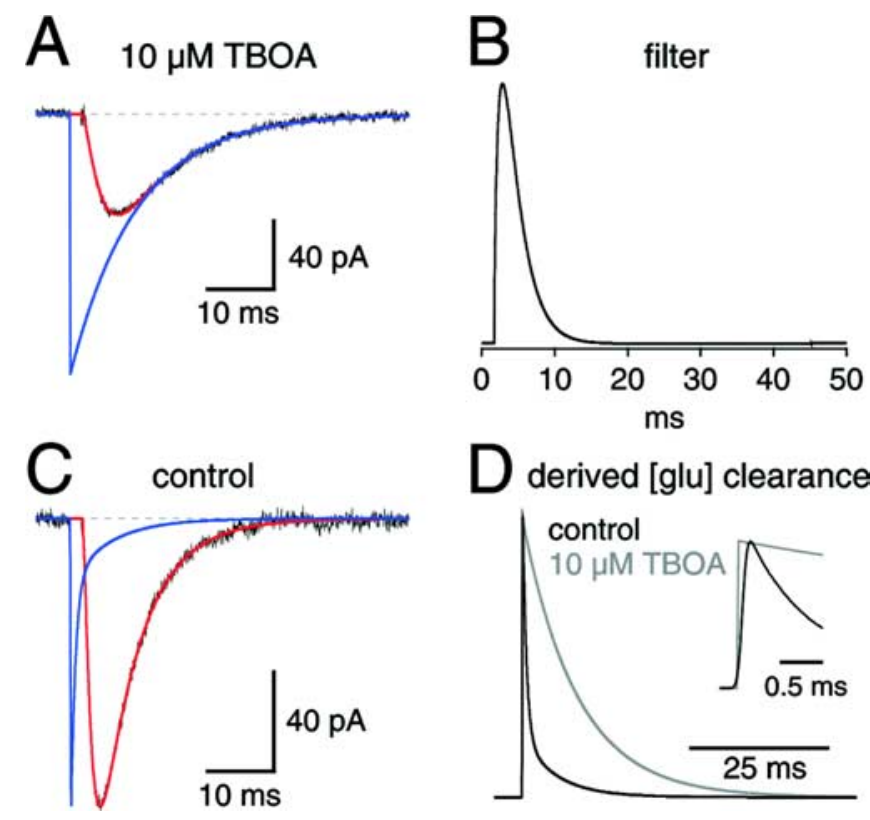

$\mathrm{D}$ derived [glu] clearance

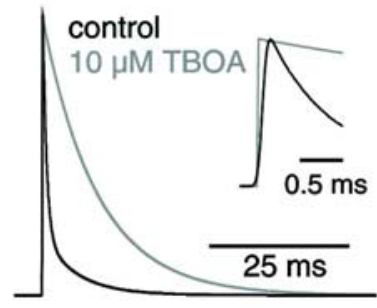

Figure 6. Deconvolving the filter to estimate glutamate clearance time in an adult astrocyte. $A$, STC recorded in the presence of $10 \mu \mathrm{M} \mathrm{TBOA}$ (black trace) and fit by a continuous function (red trace; see Materials and Methods). The STC decay was fit by a single-exponential function that was taken to indicate the slowed glutamate clearance rate in the presence of transporter antagonist (blue trace). $\boldsymbol{B}$, Temporal characteristics of filter derived from response in $\boldsymbol{A}$ by deconvolving the exponential clearance time course from the continuous fit to the STC in $10 \mu \mathrm{M}$ TBOA. C, STC recorded from the same astrocyte as in $\boldsymbol{A}$ in control conditions (black trace) and fit by a continuous function (red trace). The filter in $\boldsymbol{B}$ was deconvolved from the continuous function to derive an estimate of glutamate clearance in control conditions (blue trace). $\boldsymbol{D}$, Derived glutamate clearance time courses in control (black trace) and TBOA (gray trace), normalized and superimposed. Inset, Same traces on an expanded time scale for comparison of rising phases.

contributes insignificantly to the time course of the STC (see Discussion).

In five of the seven cells described above, deconvolution of the derived clearance rate from the STC in $10 \mu \mathrm{M}$ TBOA yielded a biphasic waveform (Fig. $5 B$ ). In the two remaining cells, the resulting waveform contained oscillations caused by the deconvolution, so these cells were excluded from further analysis. The biphasic waveform, representing the temporal characteristics of the rest of the system, exhibited a $20-80 \%$ rise time of $1.0 \pm 0.5$ $\mathrm{ms}$ and a decay rate of $3.9 \pm 2.0 \mathrm{~ms}(n=5)$ (Fig. $4 B)$. To estimate the glutamate clearance time course with uptake fully intact, this "filter" waveform was deconvolved from the STC recorded under control conditions (Fig. 4C). This resulted in a clearance time course waveform that rose rapidly $(20-80 \%$ rise time, $0.61 \pm$ $0.060 \mathrm{~ms})$ and decayed exponentially $(\tau=5.8 \pm 1.2 \mathrm{~ms} ; n=5)$ (Fig. $4 D$ ). The decay of the derived clearance waveform was significantly faster than STC decay $\left(\tau_{\text {STC }}, 10.2 \pm 1.6 \mathrm{~ms} ; n=5 ; p \ll\right.$ 0.001 ), indicating that the STC in young animals constitutes a significantly filtered representation of glutamate clearance.

Successful deconvolutions were performed in five adult astrocytes; in these cells, the STC in $10 \mu \mathrm{M}$ TBOA decayed with an exponential time constant of $10.5 \pm 0.78 \mathrm{~ms}(n=5)$ (Fig. $6 A)$. The deconvolved filter exhibited a $20-80 \%$ rise time of $0.5 \pm 0.2$ $\mathrm{ms}$ and a decay time constant of $2.8 \pm 0.9 \mathrm{~ms}$ (Fig. $6 \mathrm{~B}$ ). The derived clearance waveform also rose and decayed more rapidly $\left(20-80 \%\right.$ rise time, $\left.0.16 \pm 0.09 \mathrm{~ms} ; \tau_{\text {decay }}, 0.75 \pm 0.15 \mathrm{~ms}\right)$ in adult astrocytes compared with P12-P14 astrocytes (rise time, $\left.p \ll 0.001 ; \tau_{\text {decay }}, p<0.001\right)$. The derived control clearance time course in adult astrocytes was eight times faster than the STC decay in the same cells $\left(\tau_{\mathrm{STC}}, 6.17 \pm 0.95 \mathrm{~ms} ; n=5 ; p<0.001\right)$. 


\section{Another way to measure clearance time}

The preceding deconvolution analysis indicates that adult astrocytes take up synaptically released glutamate faster than P12-P14 astrocytes and that in the adult hippocampus, glutamate is taken up within $1 \mathrm{~ms}$ after its synaptic release. To confirm these results, a different analytical approach was taken that exploits the apparent linearity of the system but uses a different measure of the STC time course. This approach relies on the fact that centroids add under convolution (Bracewell, 2000) [i.e., for a function $c(t)$ that is the convolution of two other functions, $a(t)$ and $b(t),\langle t\rangle_{\mathrm{c}}=$ $\langle t\rangle_{\mathrm{a}}+\langle t\rangle_{\mathrm{b}}$ ]. If the STC is a linearly filtered representation of glutamate clearance, then $\langle t\rangle_{\text {STC }}=\langle t\rangle_{\text {clearance }}+\langle t\rangle_{\text {filter }}$.

In a linear system, the rate of clearance is proportional to the concentration of glutamate transporters $([T])$. Consequently, the inverse of the clearance rate $\left(\langle t\rangle_{\text {clearance }}\right)$ is linearly related to the inverse of the transporter concentration, $1 /[T]$. In the hypothetical case of instantaneous binding $\left(1 /[T]=0,\langle t\rangle_{\text {clearance }}=\right.$ $0),\langle t\rangle_{\text {STC }}$ would reflect the filter characteristics. On a plot of $<t>_{\mathrm{STC}}$ versus $1 /[T]$ (normalized such that $[T]_{\mathrm{control}}=1$ ), then the intercept would represent $\langle t\rangle_{\text {filter }}$ and the slope would reflect $\langle t\rangle_{\text {clearance }}$ (Fig. 7C).

Relative changes in $[T]$ caused by TBOA were calculated by measuring the initial slope of the STC to obtain a relative measure of response amplitude while minimizing distortion by the filter (Fig. $7 A, B$ ). This approach appears to provide a good approximation of relative uptake capacity: dihydrokainate (DHK), which blocks the GLT-1 transporter subtype specifically and completely at the concentration used $(300 \mu \mathrm{M})$, reduced the initial slope of STCs in adult astrocytes by $79.2 \pm 4.8 \%$ of control $(n=4$; data not shown), consistent with quantitative immunoblotting studies showing that GLT-1 constitutes $80 \%$ of the astroglial transporters in the adult hippocampus (Lehre and Danbolt, 1998). Although the initial slope of the STC may be contaminated by release asynchrony (Fig. $4 \mathrm{~A}$ ), the ratio of initial STC slopes in TBOA and control was not significantly different from the ratio of FTC slopes recorded in the same cells $(n=4 ; p=0.88$; data not shown). In the experiments below, then, $1 /[T]$ was determined by calculating the ratio of the STC initial slope in control to that in the presence of antagonist.

The control/TBOA initial slope ratio in P12-P14 astrocytes $(5.37 \pm 1.02 ; n=7)$ was not significantly different from that in adult astrocytes $(4.60 \pm 1.48 ; n=8 ; p=0.15)$. The relationship between $\langle t\rangle_{\text {STC }}$ and $1 /[T]$, however, was shallower in adult astrocytes than in P12-P14 astrocytes (Fig. 7C), consistent with the conclusion from deconvolution analysis that clearance is faster in the adult. The derived $\langle t\rangle\rangle_{\text {clearance }}$ in adult cells was quite similar to $\tau_{\text {clearance }}$ derived via deconvolution $(1.0 \pm 0.2$ vs $0.75 \pm 0.15$ $\mathrm{ms})$, although $\langle t\rangle_{\text {clearance }}$ was somewhat less than $\tau_{\text {clearance }}$ derived in P12-P14 cells ( $3.6 \pm 0.4$ vs $5.78 \pm 1.22 \mathrm{~ms})$. The centroid analysis also indicated that the filter in adult astrocytes $\left(\langle t\rangle_{\text {filter }}\right.$, $5.75 \pm 0.68 \mathrm{~ms}$ ) exhibited faster temporal characteristics than in $\mathrm{P} 12-\mathrm{P} 14$ astrocytes $\left.(<\mathrm{t}\rangle_{\text {filter }}, 13.4 \pm 1.5 \mathrm{~ms}\right)$. This result is qualitatively similar to that obtained from deconvolution, although the deconvolution approach predicted faster filter characteristics.

\section{Relating the time course of clearance to the distance of diffusion}

The experiments described above indicate that glutamate is taken up by glial transporters in the juvenile (P12-P14) hippocampus within $4-6 \mathrm{~ms}$ and within $\sim 1 \mathrm{~ms}$ in the adult, suggesting that synaptically released transmitter is permitted to diffuse further in younger animals. To derive a quantitative estimate of diffusion distance, a Monte Carlo approach was taken to simulate the ran-
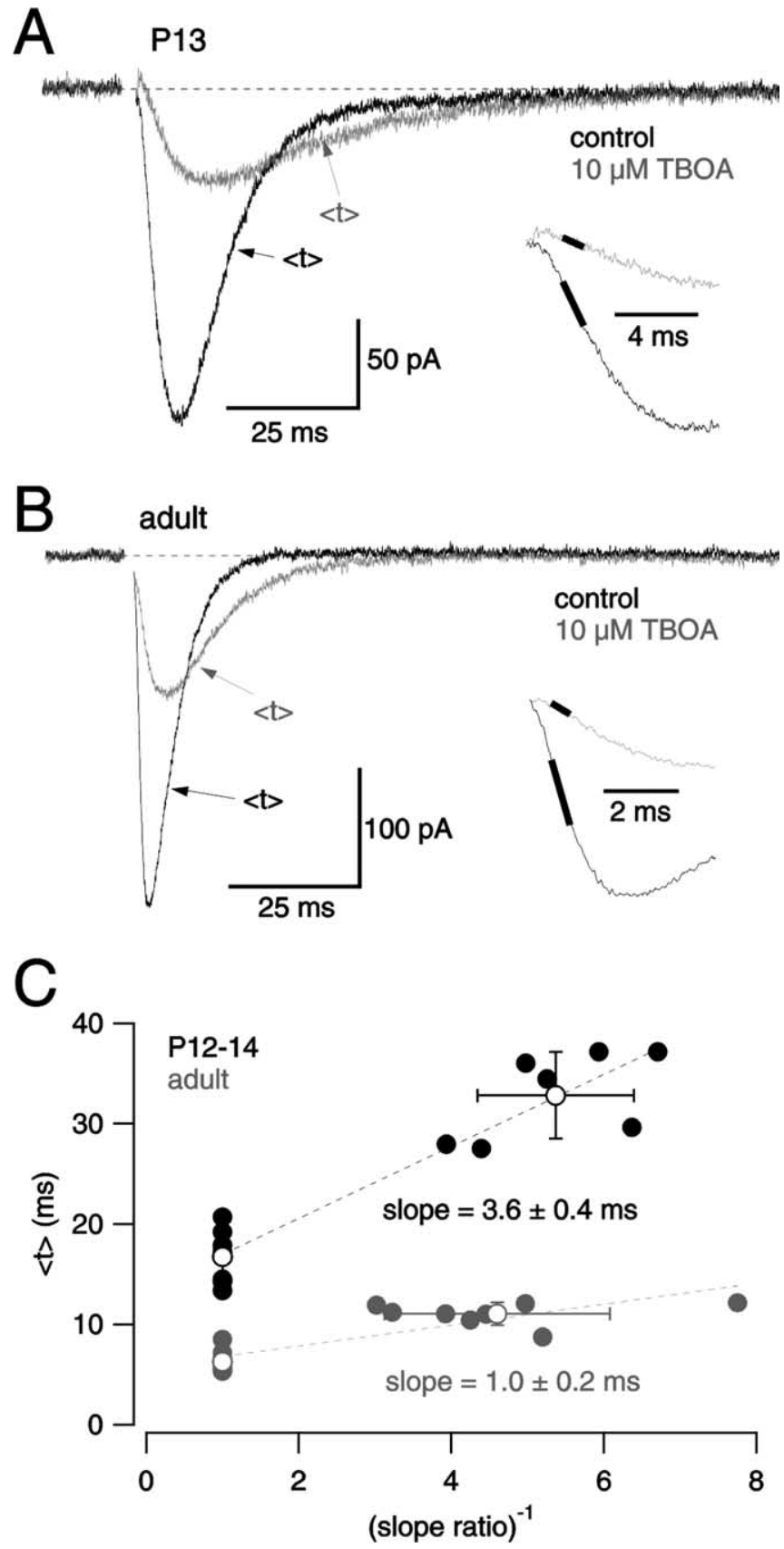

Figure 7. Deriving glutamate clearance time from analysis of centroids. $A$, STCs recorded from a P13 astrocyte in control (black trace) and in the presence of $10 \mu \mathrm{M} \mathrm{TBOA} \mathrm{(gray} \mathrm{trace).} \mathrm{The}$ centroid of each STC is indicated by arrows. Inset, Linear fits (thick black lines) to rising phases were used to quantify reduction in uptake capacity. $\boldsymbol{B}$, As in $\boldsymbol{A}$, but responses were recorded in an adult astrocyte. $C$, Centroid of STC plotted versus inverse STC slope ratio for P12-P14 astrocytes (black symbols; $n=7$ ) and adult astrocytes (gray symbols; $n=8$ ). The slope in each case reflects the centroid of glutamate clearance (see Results for details).

dom walk of 5000 individual glutamate molecules from a point source (see Materials and Methods). These stochastic simulations mimicked analytical solutions for diffusion in a disk or sphere (Barbour and Hausser, 1997) (Fig. 8 A). Synaptic release was simulated by locating the point source at the center of a $320-\mathrm{nm}$ diameter disk (Fig. 8A, inset), to emulate the average boutonspine contact at CA1 synapses (Ventura and Harris, 1999). The extrasynaptic space was modeled as a tortuous, isotropic medium (Rusakov and Kullmann, 1998) containing a variable concentration of glutamate transporters (EAAT2) (Bergles et al., 2002). 

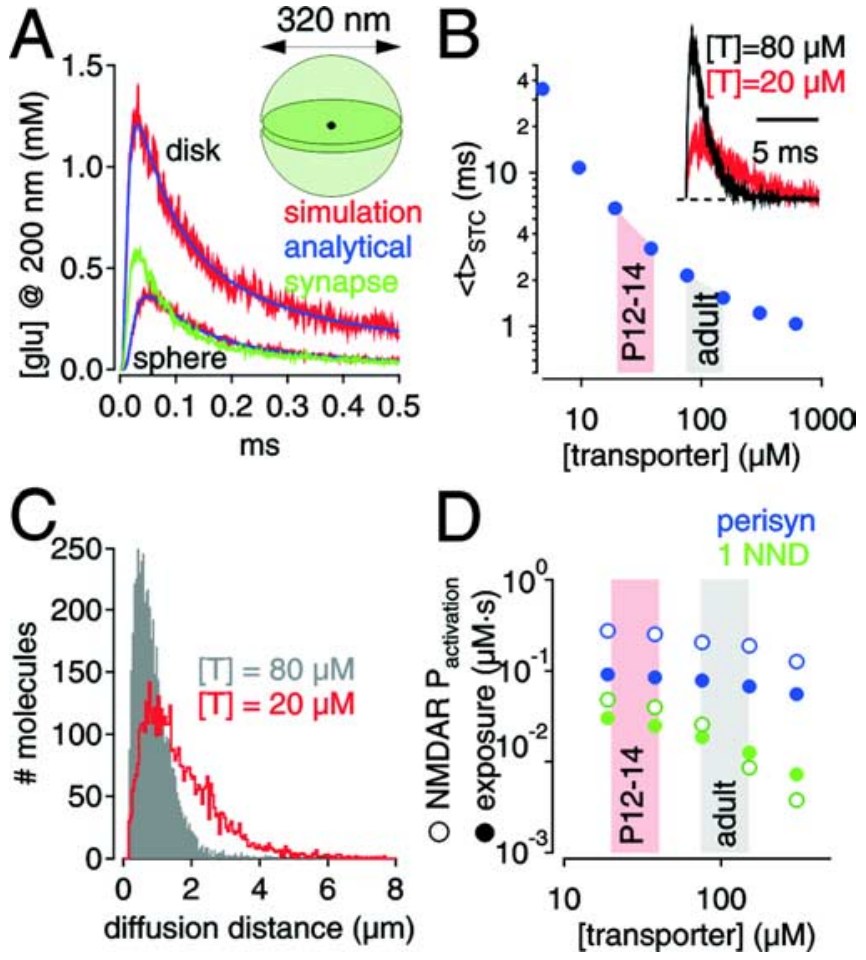

Figure 8. Monte Carlo diffusion model to simulate dynamics of glutamate diffusion and uptake. $\boldsymbol{A}$, Monte Carlo-simulated diffusion matches theoretical diffusion equations. Simulated diffusion (red traces) into an "infinite" disk or three-dimensional space (sphere) matched well theoretical equations [adapted from Barbour and Hausser (1997)]. [glu] was calculated $200 \mathrm{~nm}$ from the point release of 5000 glutamate molecules (see Materials and Methods for simulation parameters). In subsequent simulations, release occurred at the center of a 320-nm-diameter disk (inset) to simulate the average dimension of a synaptic contact in CA1 (Ventura and Harris, 1999). The simulated [glu] waveform measured $40 \mathrm{~nm}$ outside the synapse ( $200 \mathrm{~nm}$ from the release site; green trace) reflected characteristics of the disk and sphere simulations. $\boldsymbol{B}$, Centroid of simulated STCs decreased as transporter concentration increased. Inset, Example traces of simulated STCs. Transporter kinetics were represented by a modified kinetic model of EAAT2 (Bergles et al., 2002) (see Materials and Methods). Traces are averages of five simulations. $\boldsymbol{C}$, Histograms showing the radial distance from the release site at which each glutamate molecule is taken up during simulations involving two different transporter concentrations. D, Transporters limit activation of extrasynaptic receptors. Glutamate exposure (closed circles) was calculated at $220 \mathrm{~nm}$ [perisynaptic (perisyn)] and $465 \mathrm{~nm}$ [nearest-neighbor distance (NND)] (Rusakov and Kullmann, 1998) by integrating the glutamate concentration waveform. NMDAR activation probability (open circles) was determined by driving an NMDAR kinetic model (Lester and Jahr, 1992) with glutamate concentration waveforms generated by the diffusion simulations (see Materials and Methods). For comparison, when the simulated [glutamate] waveform within the synaptic cleft was used to drive the NMDAR model, $P_{\text {activation }}$ was 0.48 .

Transporters were excluded from the $320 \mathrm{~nm}$ synaptic disk and were not modeled explicitly until bound by glutamate (see Materials and Methods).

Transmitter release from within the simulated synapse elicited STCs that grew faster as transporter concentration $([T])$ was increased (Fig. $8 B$ ). At high values of $[T],\langle t\rangle_{\text {STC }}$ became limited by the imperfect efficiency of transporters, [i.e., glutamate often unbinds (with a time constant of $\sim 1 \mathrm{~ms}$ ) instead of being transported] (Bergles et al., 2002). Consequently, when [T]>150 $\mu \mathrm{M}$, large changes in transporter efficacy exerted relatively small effects on simulated clearance time. The low value of $\langle t\rangle$ clearance measured in adult astrocytes $(\sim 1 \mathrm{~ms})$ suggests that the time course of clearance may be limited by transporter efficiency. However, blocking $\sim 80 \%$ of the transporters with either $10 \mu \mathrm{M}$ TBOA (Fig. 6) or $300 \mu \mathrm{M}$ DHK (data not shown) increased the experimental $\langle t\rangle_{\mathrm{STC}}$ severalfold, suggesting adult CA1 neuropil astrocytes probably express transporters in the range of 75-150 $\mu \mathrm{M}$. This corresponds to an astroglial surface membrane concentration of $6500-13,000 \mu \mathrm{m}^{-2}$, well within the range of quantitative immunoblotting measurements $\left(10,800 \mu \mathrm{m}^{-2}\right.$ ) (Lehre and Danbolt, 1998). The relationship between simulated $\langle t\rangle_{\text {STC }}$ and $[T]$ suggests that the juvenile CA1 neuropil, in which extracellular glutamate is cleared in 4-6 ms, expresses functional transporters at an effective concentration of $20-40 \mu \mathrm{M}$, corresponding to an astroglial surface membrane density of $1700-3400 \mu \mathrm{m}^{-2}$ (Lehre and Danbolt, 1998). This value corresponds well to the transporter density estimated in membrane patches excised from CA1 astrocytes of the same age $\left(2500 \mu \mathrm{m}^{-2}\right)$ (Bergles and Jahr, 1997).

Lower transporter capacity allowed released glutamate to diffuse further before being taken up (Fig. $8 C$ ). When $[T]=80 \mu \mathrm{M}$, corresponding approximately to the experimentally observed clearance rate in adult astrocytes, glutamate diffused $0.89 \pm 0.58$ $\mu \mathrm{m}$ (linear distance, $n=5000$ glutamate molecules released) from the point of release before being taken up. When $[T]$ was reduced to $20 \mu \mathrm{M}$ to simulate the clearance rate observed in P12P14 astrocytes, glutamate diffused twice as far, on average (1.7 \pm $1.2 \mu \mathrm{m})$. Consequently, the time integral of glutamate concentration (Fig. $8 D$, exposure) was highest at low values of $[T]$, particularly in regions more distant from the site of release (e.g., 465 $\mathrm{nm}$, the mean nearest-neighbor distance between excitatory synapses in CA1) (Rusakov and Kullmann, 1998) (Fig. 8D). Differences in exposure were amplified by NMDARs, which require multiple binding events for activation (Clements and Westbrook, 1991; Lester and Jahr, 1992) (Fig. 8D). A comparison of extrasynaptic NMDAR activation at different ages would have to account for a likely developmental decrease of $\sim 20 \%$ in the extracellular volume fraction between P12-P14 and adult (Vorisek and Sykova, 1997; Sykova, 2004). Consequently, extrasynaptic [glu] in young neuropil may be $\sim 20 \%$ lower than indicated in Figure $8 D$.

\section{Developmental changes in NMDAR activation by glutamate spillover}

The simulations presented above suggest that transmitter dilution by diffusion reduces extrasynaptic NMDAR activation by glutamate spillover (Barbour, 2001). In adult neuropil, dilution combined with avid uptake would appear to make NMDAR activation in neighboring synapses extremely rare $(\sim 1 \%)$ (Fig. $8 D$ ), although activation of more proximal perisynaptic receptors may be more likely. In younger tissue, lower uptake capacity may allow for greater activation of extrasynaptic receptors (Fig. $8 D$ ), the aforementioned changes in extracellular volume fraction notwithstanding. If extrasynaptic receptor activation is greatest earlier in development, reducing uptake with $10 \mu \mathrm{M}$ TBOA would have a larger effect on NMDAR EPSCs in younger slices, provided that blocking uptake preferentially enhances the spillover component of the EPSC (Asztely et al., 1997; Diamond, 2001; Arnth-Jensen et al., 2002).

To test this idea, NMDAR EPSCs were recorded at positive potentials (CPP and MK-801 were omitted from the superfusion solution), and the effect of $10 \mu \mathrm{M}$ TBOA was tested (Fig. 9). In P10-P14 slices, TBOA significantly prolonged the half-decay time of the NMDAR EPSC ( to $183 \pm 21 \%$ of control; $n=4 ; p=$ 0.011 ) (Fig. $9 A, B, E, F$ ), with no significant effect on the peak $(107 \pm 11 \%$ of control; $n=4 ; p=0.24)$. In adult slices, TBOA had small, yet consistent, effects on both the peak (127 $\pm 16 \%$ of control; $n=6 ; p=0.020$ ) (Fig. 9C-F) and the half-decay (127 \pm $19 \%$ of control; $n=6 ; p=0.22)$ of the EPSC. There were no significant differences between age groups in EPSC amplitude 

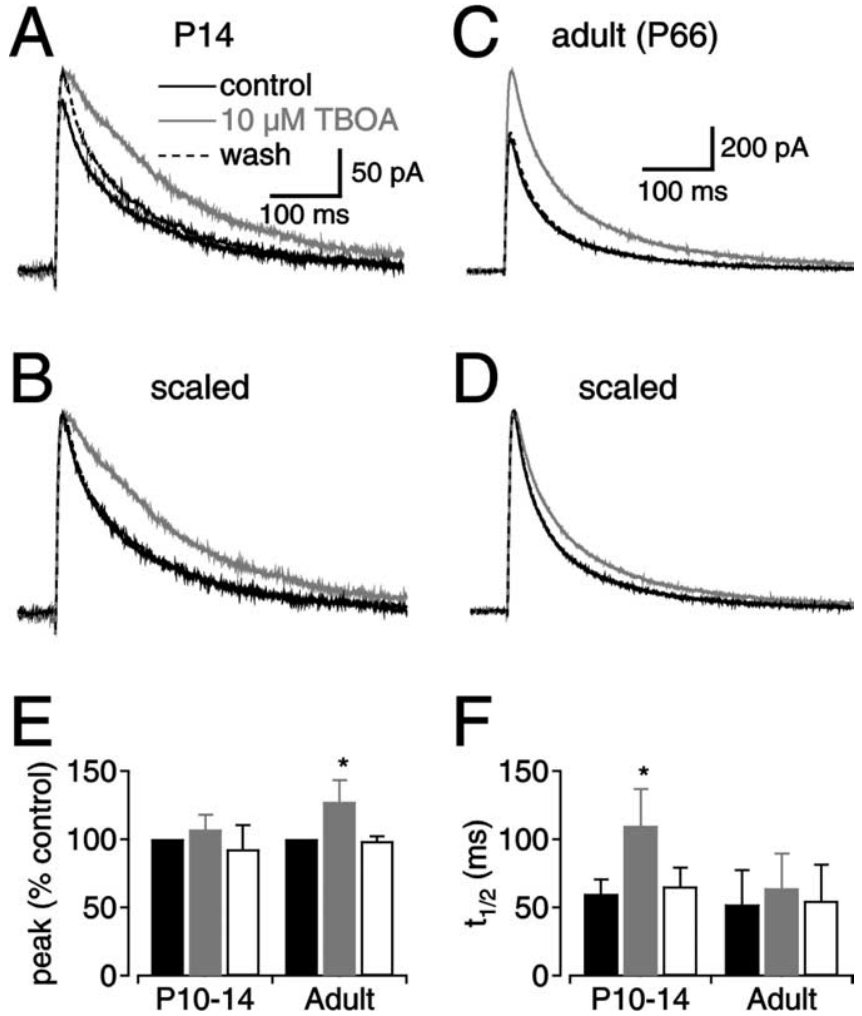

Figure 9. Effects of $10 \mu \mathrm{m}$ TBOA on NMDAR EPSCS. A, EPSCs recorded from a P14 CA1 pyramidal cell in control (black), in the presence of $10 \mu \mathrm{m} \mathrm{TBOA} \mathrm{(gray),} \mathrm{and} \mathrm{after} \mathrm{washout} \mathrm{(wash)} \mathrm{of}$ TBOA (black dotted line). $V_{\text {hold }}=+30 \mathrm{mV}$. B, Traces in $A$ scaled to the same amplitude. C, As in $\boldsymbol{A}$ but recorded from an adult (P66) pyramidal cell. $\boldsymbol{D}$, Traces in $($ scaled to the same amplitude. $\boldsymbol{E}$, Summary effects of $10 \mu \mathrm{m}$ TBOA on NMDAR EPSC peak amplitude in P10-P14 $(n=4)$ and adult $(n=6)$ pyramidal cells. $\boldsymbol{F}$, Summary effects of $10 \mu \mathrm{m}$ TBOA on NMDAR EPSC half-decay time in P10 P14 $(n=4)$ and adult $(n=6)$ pyramidal cells. The bars in $\boldsymbol{E}$ and $\boldsymbol{F}$ correspond to control (black), $10 \mu \mathrm{M} \mathrm{TBOA} \mathrm{(gray),} \mathrm{and} \mathrm{wash} \mathrm{(white)} \mathrm{conditions.} \mathrm{Asterisks} \mathrm{indicate} \mathrm{a} \mathrm{statisti-}$ cally significant difference $(p<0.05)$ compared with control.

$(\mathrm{P} 12,372 \pm 258 \mathrm{pA}$; adult, $504 \pm 121 \mathrm{pA} ; p=0.39)$ or half-decay time $(\mathrm{P} 12,60 \pm 11 \mathrm{~ms}$; adult, $52 \pm 26 \mathrm{~ms} ; p=0.53)$. These results suggest that extrasynaptic receptors constitute a greater fraction of the NMDARs activated during EPSCs in younger slices.

\section{Discussion}

The results presented here indicate that CA1 astroglial glutamate transporters take up synaptically released neurotransmitter very rapidly, even faster than indicated by the STC time course and nearly as fast as uptake by postsynaptic transporters located within the cleft of climbing fiber synapses on cerebellar Purkinje neurons (Auger and Attwell, 2000). A developmental increase in glutamate clearance rate in CA1 neuropil corresponds to a concomitant decrease in extrasynaptic NMDAR activation during an EPSC. Consequently, receptors located outside active synapses, in quiescent neighboring synapses or, more likely, in the dendritic membranes between synapses, may play particularly important roles in the nascent hippocampal network.

\section{Mechanisms underlying the STC time course in CA1}

The approach used here to estimate the glutamate clearance rate requires that the STC is a linear indicator of the total amount of extracellular glutamate. This is supported by evidence that STC amplitude varies proportionally with stimulus strength (Fig. $3 \mathrm{~A}$ ) (Diamond and Jahr, 2000) and that the STC and FTC waveforms remained invariant over broad changes in amplitude (Fig. $3 D$ ).
Consequently, the STC offers significant analytical advantages over neuronal synaptic currents, which are mediated by nonlinear, multiliganded glutamate receptors exposed to high transmitter concentrations within spatially restricted synapses located in active dendrites at varying electrotonic locations from the somatic recording electrode.

The linear characteristics of the STC likely reflect specific properties of astrocytes, the CA1 neuropil, and glutamate transporters. Very fine astrocytic processes form a dense, evenly distributed mesh throughout the CA1 neuropil (Bushong et al., 2002), suggesting that glutamate encounters a consistent density of astrocyte membranes throughout its extrasynaptic diffusion lifetime, although clusters of transporters in glial membranes could indicate spatially inhomogeneous uptake capacity (Minelli et al., 2001; Zhou and Sutherland, 2004). Transporters clustered around synapses could restrict glutamate diffusion by acting as rapid buffers (Diamond and Jahr, 1997) but they do not appear to speed clearance, because synaptically released glutamate is taken up at the same rate as glutamate released throughout the neuropil by flash photolysis (Fig. 4). This apparent contradiction may be explained by kinetic properties of the transporters, which transport and unbind glutamate with similar probability and with a time constant of $\sim 1 \mathrm{~ms}$ (Bergles et al., 2002). This imperfect efficiency may prove to be the rate-limiting step in clearance, at least in the adult, rendering submicrometer spatial inhomogeneities in transporter expression insignificant in determining the time course of uptake.

The similarity between the FTC and STC suggests that the dominant mechanism distorting the STC time course is the electrotonic properties of the astrocytic membrane. The passive cell membrane constitutes a linear, low-pass filter that slows the signal reaching the soma (relative to the actual synaptic conductance) by an amount that is independent of the size of the conductance (Rall, 1989). In neurons, however, depolarizations in the dendrites can activate voltage-gated dendritic conductances that distort the synaptic signal waveform in a nonlinear manner. As a result, EPSC waveforms can become slower with stronger stimulation attributable solely to imperfect space clamp. STCs do not exhibit such nonlinearity (Fig. 3), probably because any voltage-gated conductances in astrocyte processes are very small compared with the large potassium leak. Although the astrocyte membrane resistance appears very low, the dense mesh of fine processes (Bushong et al., 2002) likely constitutes a significant capacitance that may give rise to a membrane time constant that is sufficient to distort transporter-mediated conductances.

\section{Potential sources of error}

The experiments described here were performed with low concentrations of sodium $(0.2 \mathrm{~mm})$ and glutamate $(0 \mathrm{~mm})$ in the patch electrode to deter reversal of the transporter cycle. Transporter efficiency, and therefore the rate of glutamate clearance, would decrease if $\left[\mathrm{Na}^{+}\right]_{\mathrm{i}}$ and $[\mathrm{glu}]_{\mathrm{i}}$ were higher, but the model used here (Fig. 8) suggests that such decreases would be relatively minor. When $\left[\mathrm{Na}^{+}\right]_{\mathrm{i}}$ was set to the resting value measured in cultured mouse cortical astrocytes (10 mM) (Chatton et al., 2000), the glutamate clearance time course was similar to that shown in Figure 8 and remained constant $(<5 \%$ change) over a wide range of $[\mathrm{glu}]_{\mathrm{i}}\left(0.05-5 \mathrm{mM}\right.$; data not shown). When $\left[\mathrm{Na}^{+}\right]_{\mathrm{i}}=$ $30 \mathrm{~mm}$ (and $\left.[\mathrm{glu}]_{\mathrm{i}}=5 \mathrm{~mm}\right)$, simulating prolonged, very high extracellular glutamate levels ( $1 \mathrm{~mm}$ for $>1 \mathrm{~min}$ ) (Chatton et al., 2000 ), simulated clearance was slowed by $20 \%$. Under these conditions, however, the average diffusion distance of synaptically released glutamate increased only $4 \%$, presumably because trans- 
porters operating as exchangers are available to buffer extracellular glutamate diffusion. Thus, the experimental and simulation parameters used here appear to constitute reasonable approximations of physiological conditions.

One key assumption in the deconvolution and centroid analysis is that the temporal characteristics of the filter remain the same in control conditions and in the presence of $10 \mu \mathrm{M}$ TBOA. If the filter reflects the electrotonic properties of the astrocytic membrane, this is probably a reasonable assumption, because TBOA does not induce detectable changes in input resistance. Another potentially confounding possibility is that the complement of transporters in $10 \mu \mathrm{M}$ TBOA could have different kinetic properties than those in control. Hippocampal glial transporters comprise the EAAT1 (GLAST) and EAAT2 (GLT-1) subtypes (Rothstein et al., 1994), of which $\sim 80 \%$ is EAAT2 (Lehre and Danbolt, 1998). TBOA exhibits a greater affinity for EAAT2 $\left(\mathrm{IC}_{50}, 5.5 \mu \mathrm{M}\right)$ than for EAAT1 $\left(\mathrm{IC}_{50}, 67 \mu \mathrm{M}\right)$ (Shimamoto et al., 1998), so a TBOA concentration sufficient to block $75-80 \%$ of the transporters (estimated by the reduction in initial slope) would block $85-90 \%$ of EAAT2 and $\sim 35 \%$ of EAAT1 (Shimamoto et al., 1998), leaving a relatively even mix of the two subtypes. However, the time course of the STC in $10 \mu \mathrm{M}$ TBOA $(<t>=11.1 \pm 1.1 \mathrm{~ms} ; n=8)$ was not significantly different from that in $300 \mu \mathrm{M} \mathrm{DHK}(<t>=9.9 \pm 2.2 \mathrm{~ms} ; n=4 ; p=0.32)$, which blocks EAAT2 completely, leaving essentially 100\% EAAT1 (Arriza et al., 1994). Changing the relative contributions of the two glial transporter subtypes therefore appears to have little effect on the STC time course.

Three features of the deconvolution analysis (Figs. 5, 6) could distort the time course of the derived filter and, consequently, the estimated glutamate clearance time course in control conditions. First, the STC decay in the presence of $10 \mu \mathrm{M}$ TBOA is taken to indicate directly the slowed clearance time course, because the decay is twofold to threefold slower than in control. If, because of small effects of the filter, the decay in TBOA were slower than clearance, the actual filter characteristics would be slower than calculated. Consequently, the rate of glutamate clearance in control conditions would be faster than estimated. Second, representing the clearance time course in TBOA with a monoexponential fit of the STC decay may neglect faster components of clearance that are obscured by the filter and do not contribute to the decay. Third, for the sake of simplicity, the rising phase of the exponential clearance time course in $10 \mu \mathrm{M}$ TBOA was made instantaneous (Figs. 5A, 6A). This is unlikely to be the case, and any slowing in the rise time of the actual clearance time course in TBOA would make the filter slightly faster, and clearance in control conditions slightly slower, than estimated. The centroid approach is vulnerable to none of these problems, however, and the similar clearance times estimated from the two approaches support the simplifications used in the deconvolution approach.

\section{Developmental regulation of glutamate diffusion and extrasynaptic receptor activation}

Glutamate receptor targeting and expression play critical roles in the function and developmental maturation of excitatory synapses. For example, NMDARs, which mediate several different forms of activity-dependent synaptic plasticity (Malenka and Nicoll, 1993), change in subunit composition and synaptic localization during development. In general, NMDARs containing NR2B subunits predominate early in development, when many receptors are located extrasynaptically before the formation of nascent synapses; later in development, a greater fraction of NMDARs contain NR2A subunits and are located within synap- tic contacts (for review, see Perez-Otano and Ehlers, 2004). In addition to their contrasting developmental time courses and targeting characteristics, NR2A- and NR2B-containing receptors exhibit distinct biophysical properties: NR2B-containing receptor channels are less susceptible to magnesium block, exhibit a longer open time (Monyer et al., 1992), and have a higher affinity for glutamate (Laurie and Seeburg, 1994), making them more sensitive reporters of low extrasynaptic glutamate levels. The preferential expression of extrasynaptic NR2B-containing receptors occurs when the capacity of glial glutamate uptake is relatively low (Furuta et al., 1997; Ullensvang et al., 1997) (Figs. 2, 7), a combination that would maximize postsynaptic detection of extrasynaptic glutamate. This is likely to be a crucial step in the process of synapse formation, whether glutamate released from nascent synapses recruits extrasynaptic NMDARs (Friedman et al., 2000) or whether transmitter released from orphan boutons induces synapse formation by activating distant clusters of NMDARs floating in the dendritic membrane (Washbourne et al., 2002). As synapses mature and NR2A-containing receptors are confined to the postsynaptic scaffold, increased transporter expression may decrease transmitter diffusion between synapses and increase synapse specificity, although intersynaptic cross talk may not be prohibited entirely (Asztely et al., 1997; Lozovaya et al., 1999; Arnth-Jensen et al., 2002; Scimemi et al., 2004).

\section{References}

Arnth-Jensen N, Jaboudon D, Scanziani M (2002) Cooperation between independent hippocampal synapses is controlled by glutamate uptake. Nat Neurosci 5:325-331.

Arriza JL, Fairman WA, Wadiche JI, Murdoch GH, Kavanaugh MP, Amara SG (1994) Functional comparisons of three glutamate transporter subtypes cloned from human motor cortex. J Neurosci 14:5559-5569.

Asztely F, Erdemli G, Kullmann DM (1997) Extrasynaptic glutamate spillover in the hippocampus: dependence on temperature and the role of active glutamate uptake. Neuron 18:281-293.

Auger C, Attwell D (2000) Fast removal of synaptic glutamate by postsynaptic transporters. Neuron 28:547-558.

Barbour B (2001) An evaluation of synapse independence. J Neurosci 21:7969-7984

Barbour B, Hausser M (1997) Intersynaptic diffusion of neurotransmitter. Trends Neurosci 20:377-384.

Bergles DE, Jahr CE (1997) Synaptic activation of glutamate transporters in hippocampal astrocytes. Neuron 19:1297-1308.

Bergles DE, Jahr CE (1998) Glial contribution to glutamate uptake at Schaffer collateral-commissural synapses in the hippocampus. J Neurosci 18:7709-7716.

Bergles DE, Diamond JS, Jahr CE (1999) Clearance of glutamate inside the synapse and beyond. Curr Opin Neurobiol 9:293-298.

Bergles DE, Tzingounis AV, Jahr CE (2002) Comparison of coupled and uncoupled currents during glutamate uptake by GLT-1 transporters. J Neurosci 22:10153-10162.

Bracewell R (2000) The Fourier Transform and its applications, Ed 3. Boston: McGraw-Hill.

Bushong EA, Martone ME, Jones YZ, Ellisman MH (2002) Protoplasmic astrocytes in CA1 stratum radiatum occupy separate anatomical domains. J Neurosci 22:183-192.

Chatton JY, Marquet P, Magistretti PJ (2000) A quantitative analysis of L-glutamate-regulated $\mathrm{Na}^{+}$dynamics in mouse cortical astrocytes: implications for cellular bioenergetics. Eur J Neurosci 12:3843-3853.

Chaudhry FA, Lehre KP, van Lookeren Campagne M, Ottersen OP, Danbolt NC, Storm-Mathisen J (1995) Glutamate transporters in glial plasma membranes: highly differentiated localizations revealed by quantitative ultrastructural immunocytochemistry. Neuron 15:711-720.

Clements JD, Westbrook GL (1991) Activation kinetics reveal the number of glutamate and glycine binding sites on the $N$-methyl-D-aspartate receptor. Neuron 7:605-613.

Diamond JS (2001) Neuronal glutamate transporters limit activation of NMDA receptors by neurotransmitter spillover on CA1 pyramidal cells. J Neurosci 21:8328-8338. 
Diamond JS, Jahr CE (1997) Transporters buffer synaptically released glutamate on a submillisecond time scale. J Neurosci 17:4672-4687.

Diamond JS, Jahr CE (2000) Synaptically released glutamate does not overwhelm transporters on hippocampal astrocytes during high-frequency stimulation. J Neurophysiol 83:2835-2843.

Friedman HV, Bresler T, Garner CC, Ziv NE (2000) Assembly of new individual excitatory synapses: time course and temporal order of synaptic molecule recruitment. Neuron 27:57-69.

Furuta A, Rothstein JD, Martin LJ (1997) Glutamate transporter protein subtypes are expressed differentially during rat CNS development. J Neurosci 17:8363-8375.

Hille B (1984) Ionic channels of excitable membranes, Ed 1. Sunderland, MA: Sinauer Associates.

Laurie DJ, Seeburg PH (1994) Ligand affinities at recombinant N-methylD-aspartate receptors depend on subunit composition. Eur J Pharmacol 268:335-345.

Lehre KP, Danbolt NC (1998) The number of glutamate transporter subtype molecules at glutamatergic synapses: chemical and stereological quantification in young adult rat brain. J Neurosci 18:8751-8757.

Lester RA, Jahr CE (1992) NMDA channel behavior depends on agonist affinity. J Neurosci 12:635-643.

Longsworth L (1953) Diffusion measurements at $25^{\circ}$ of aqueous solutions of amino acids, peptides and sugars. J Am Chem Soc 75:5705-5709.

Lozovaya NA, Kopanitsa MV, Boychuk YA, Krishtal OA (1999) Enhancement of glutamate release uncovers spillover-mediated transmission by $\mathrm{N}$-methyl-D-aspartate receptors in the rat hippocampus. Neuroscience 91:1321-1330.

Malenka RC, Nicoll RA (1993) NMDA-receptor-dependent synaptic plasticity: multiple forms and mechanisms. Trends Neurosci 16:521-527.

Matsui K, Jahr CE (2003) Ectopic release of synaptic vesicles. Neuron 40:1173-1183.

Minelli A, Barbaresi P, Reimer RJ, Edwards RH, Conti F (2001) The glial glutamate transporter GLT-1 is localized both in the vicinity of and at distance from axon terminals in the rat cerebral cortex. Neuroscience 108:51-59.

Monyer H, Sprengel R, Schoepfer R, Herb A, Higuchi M, Lomeli H, Burnashev N, Sakmann B, Seeburg PH (1992) Heteromeric NMDA receptors: molecular and functional distinction of subtypes. Science 256:1217-1221.

Nielsen TA, DiGregorio DA, Silver RA (2004) Modulation of glutamate mobility reveals the mechanism underlying slow-rising AMPAR EPSCs and the diffusion coefficient in the synaptic cleft. Neuron 42:757-771.
Perez-Otano I, Ehlers MD (2004) Learning from NMDA receptor trafficking: clues to the development and maturation of glutamatergic synapses. Neurosignals 13:175-189.

Rall W (1989) Cable theory for dendritic neurons. In: Methods in neuronal modeling: from synapses to networks, Ed 1 (Koch C, Segev I, eds), pp 9-62. Cambridge, MA: MIT.

Rothstein JD, Martin L, Levey AI, Dykes-Hoberg M, Jin L, Wu D, Nash N, Kuncl RW (1994) Localization of neuronal and glial glutamate transporters. Neuron 13:713-725.

Rusakov DA, Kullmann DM (1998) Extrasynaptic glutamate diffusion in the hippocampus: ultrastructural constraints, uptake, and receptor activation. J Neurosci 18:3158-3170.

Sakmann B, Stuart G (1995) Patch-pipette recordings from the soma, dendrites, and axon of neurons in brain slices. In: Single-channel recording, Ed 2 (Sakmann B, Neher E, eds), pp 199-211. New York: Plenum.

Scimemi A, Fine A, Kullmann DM, Rusakov DA (2004) NR2B-containing receptors mediate cross talk among hippocampal synapses. J Neurosci 24:4767-4777.

Shimamoto K, Lebrun B, Yasuda-Kamatani Y, Sakaitani M, Shigeri Y, Yumoto N, Nakajima T (1998) DL-Threo-beta-benzyloxyaspartate, a potent blocker of excitatory amino acid transporters. Mol Pharmacol 53:195-201.

Sykova E (2004) Extrasynaptic volume transmission and diffusion parameters of the extracellular space. Neuroscience 129:861-876.

Ullensvang K, Lehre KP, Storm-Mathisen J, Danbolt NC (1997) Differential developmental expression of the two rat brain glutamate transporter proteins GLAST and GLT. Eur J Neurosci 9:1646-1655.

Ventura R, Harris KM (1999) Three-dimensional relationships between hippocampal synapses and astrocytes. J Neurosci 19:6897-6906.

Vorisek I, Sykova E (1997) Evolution of anisotropic diffusion in the developing rat corpus callosum. J Neurophysiol 78:912-919.

Wadiche JI, Kavanaugh MP (1998) Macroscopic and microscopic properties of a cloned glutamate transporter/chloride channel. J Neurosci 18:7650-7661.

Washbourne P, Bennett JE, McAllister AK (2002) Rapid recruitment of NMDA receptor transport packets to nascent synapses. Nat Neurosci 5:751-759.

Zhou J, Sutherland ML (2004) Glutamate transporter cluster formation in astrocytic processes regulates glutamate uptake activity. J Neurosci 24: 6301-6306. 